\title{
Dual-polarisation reflectarray made of cells with two orthogonal sets of parallel dipoles for bandwidth and cross-polarisation improvement
}

\author{
Rafael Florencio, Jose Encinar, Rafael R. Boix, Gerardo Perez-Palomino
}

\begin{abstract}
A reflectarray antenna has been proposed to provide broadband operation, low cross-polarisation and independent beamforming in dual linear polarisation. The reflectarray element consists of two orthogonal sets of three coplanar parallel dipoles, where each set of parallel dipoles is used for controlling the phase-shift of each polarisation. A focused beam $40 \mathrm{~cm}$ reflectarray has been designed, manufactured and tested. According to the experimental results, the antenna shows a $27 \%$ bandwidth for a gain of $32.9 \mathrm{dBi}$ with a variation of $1 \mathrm{~dB}$. The antenna efficiency is better than $60 \%$ and the maximum crosspolar radiation levels are at least $30 \mathrm{~dB}$ below the main beam co-polar levels for both linear polarisations. In addition, it has been demonstrated that the antenna performance is maintained when it is designed to provide two separate beams in each linear polarisation.
\end{abstract}

\section{Introduction}

Printed reflectarray antennas are planar reflector antennas made of one or more layers of patch arrays, where the printed patches are conveniently tuned to produce a prescribed phase-shift on each element in order to collimate or to shape the radiated beam when the reflectarray is illuminated by a feed (usually a horn antenna) [1]. Printed reflectarrays present several technological advantages such as low cost and low losses when compared with phased arrays or low cross-polarisation [2] and independent beamforming [3] in dual-polarisation applications when compared with conventional reflectors.

Some of the first prototypes of reflectarray antennas were made of variable-size patches [2] printed on a grounded substrate. Single-layer reflectarrays with varying-size rectangular patches have the disadvantage that the phase range achieved was usually lower than $330^{\circ}$, and that the relation between phase-shift and patch-size was strongly non-linear, which led to narrowband behaviour $[4,5]$. These drawbacks have been overcome by the use of multilayered substrates that host either two or three metallisation levels of rectangular stacked patches [4-6] or aperture-coupled patches [7]. Several reflectarrays made of two and three layers of varying-size patches have provided very good performance in terms of bandwidth, losses and cross-polarisation for dual-polarisation applications [3, 8]. However, the use of multilayered structures involves a more complex manufacturing process derived from the bonding of different layers with printed elements.

In recent years, different broadband reflectarray elements made of multi-resonant elements printed on a single dielectric layer have been proposed to improve the bandwidth, while keeping a simple manufacturing process at the same time [9-18]. These multi-resonant elements are based on the configuration of coplanar parallel dipoles [913], concentric squared [12-14] or cross-shaped rings [15, 16], a combination of square patch and square ring slots (Phoenix cell) [17] or square loops coupled to square patches [18]. In all previous cases, the use of multiple resonances makes it possible to achieve a variation of phase-shift in a range larger than $360^{\circ}$. The dimensions of the multi-resonant elements can be adjusted in order to keep phase range larger than $360^{\circ}$, while achieving smooth and linear behaviour of the phase variation at the same time. Reflectarrays made of three coplanar parallel dipoles have shown similar performances to those made of three stacked patches in terms of bandwidth, losses and cross-polarisation [19]. However, whereas the latter can be used for dual-polarisation applications, the element based on three parallel dipoles is only suitable for single polarisation. The reflectarray elements based on concentric rings [12-16] or the Phoenix cell [17] do provide the required phasing for dual linear polarisation. However, a 4-fold rotational symmetry of the elements is assumed in most of cases in order to keep a low cross-polarisation, which imposes a 
similar phase-shift distribution in both polarisations. The 4-fold rotational symmetry can be broken, but the variations in the dimensions controlling the phase of one polarisation unavoidably modify the phase of the orthogonal polarisation as shown in [20]. The reflectarray elements based on concentric rings [12-17] or fractal patches [13] are not suitable to provide an independent phasing in each polarisation, because the phase-shift of the rings is affected by the electric field in both polarisations, unless appropriated gaps are placed in the rings to produce a polarisation selective response [21].

An independent phase control for each polarisation has been implemented in the reflectarray antennas reported in $[21,22]$, which are designed to produce the same beam for transmit and receive frequencies in $\mathrm{Ka}-$ and $\mathrm{Ku}$-bands, respectively. A single layer of rectangular patches was used in [22], which exhibits the limitations in phase response and bandwidth mentioned above. However in [21], two separate sets of coplanar open concentric rings of variable size were accommodated in the same unit cell, one for each polarisation. Although this cell configuration provides a linear phase response and broadband performance for each polarisation, the presence of the two separate sets of concentric rings in each unit cell obliges to increase the unit cell size, which should be limited by the requirement of avoiding grating lobes [13]. A dual-polarisation reflectarray cell, made of a cross-dipole and a split ring printed on the same substrate, has been proposed to provide independent phase-shift at two closely spaced frequency bands (12 and $14 \mathrm{GHz}$ ) [23]. In the past few years, several works have been reported proposing reconfigurable reflectarray cells with independent control of the reflection phase for two perpendicular linear-polarised waves using variable capacitors implemented by varactor diodes [24] or microelectromechanical system (MEMS) [25]. The narrowband performance has been overcome by proposing multi-resonant cells including several slots [26] and more complex multi-resonant geometries [27]. Although these concepts can be useful for the implementation of dual-polarisation reflectarrays with reconfigurable beams, the demonstration has been limited to the cell performance.

In some specific applications, different beams are required in each polarisation. To the best knowledge of the authors, the only reflectarray antennas implemented to produce a different beam in each polarisation are those reported in [3, 28]. In these two papers, either three layers of rectangular patches or one layer of patches are used to synthesize the desired radiation patterns. Unfortunately, the use of layers of rectangular patches leads to the aforementioned drawbacks: difficulty of fabrication in the three layers case and narrowband in the single-layer case. So far, reflectarray antenna demonstrators based on multi-resonant single-layer elements have not been reported to produce separate beams in each polarisation owing to the difficulties encountered in achieving an independent control of the two polarisations in a wide frequency band, although some preliminary results have been reported at element level including reconfigurability $[26,27]$.

In this paper, we propose a broadband multi-resonant reflectarray element for dual-polarisation applications which contains two orthogonal sets of three coplanar parallel dipoles at two different metallisation levels. Whereas one set of three coplanar parallel dipoles is used for controlling the phase-shift in one polarisation, the orthogonal set controls the phase in the orthogonal polarisation. The dipoles for the two polarisations can be printed on the opposite sides of the same dielectric layer, which should be bonded to a grounded separator. The manufacturing process of the proposed reflectarray element is substantially simpler than that of the element with three stacked rectangular patches reported in $[3,8]$ (involving at least three dielectric substrate layers and three honeycomb separators), even though it is still slightly more complex than that for single-layer elements (involving just one dielectric substrate layer). The novel element based on two orthogonal sets of parallel dipoles shares the advantages of some of the single-layer multi-resonant reflectarray elements in terms of large phase range and broadband. In addition, the element shows some additional features, such as low levels of cross-polarisation and the flexibility of providing independent adjustment of the phase for each linear polarisation. This flexibility can be used to produce a different beam in each polarisation. The proposed multi-resonant element has been used to design, manufacture and test a focused beam $40 \mathrm{~cm}$ reflectarray antenna. The experimental results obtained are in good agreement with the simulations and demonstrate a very good performance in terms of bandwidth $(27 \%$ for $1 \mathrm{~dB}$ bandwidth), low cross-polarisation (at least $30 \mathrm{~dB}$ below the co-polar maximum in a $27 \%$ bandwidth) and high efficiency (better than $60 \%$ in a $27 \%$ bandwidth). Finally, the element based on two separate sets of parallel dipoles has been used to design a reflectarray antenna that radiates two separate focused beams, one for each polarisation. The simulation results show the capabilities of the new element to provide independent control of both linear polarisations.

\section{Characterisation of reflectarray element for dual-polarisation applications}

Fig. 1 shows the reflectarray element considered in this paper. This reflectarray element consists of two orthogonal sets of three coplanar parallel dipoles. One set is introduced to control the phase-shift in one polarisation and the other set is introduced to control the phase-shift in the orthogonal polarisation, whereas the dipoles that control one of the polarisations are printed on one side of the dielectric layer of thickness $h_{2}$ and complex permittivity $\varepsilon_{2}=\varepsilon_{0} \varepsilon_{\mathrm{r} 2}\left(1-\tan \delta_{2}\right)$, the dipoles that control the orthogonal polarisation are printed on the opposite side of the same dielectric layer. The dielectric sheet where all the dipoles are printed is bonded to a grounded dielectric layer of thickness $h_{1}$ and

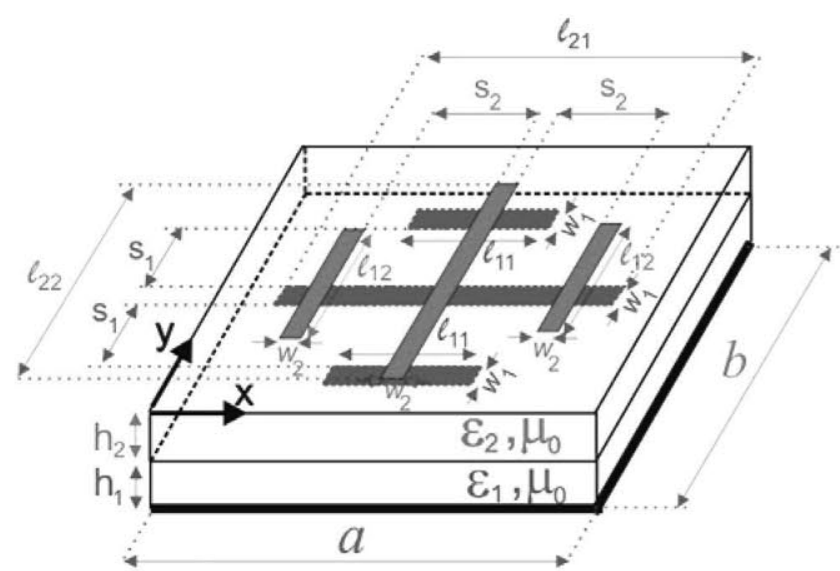

Fig. 1 Reflectarray element cell based on two orthogonal sets of three parallel dipoles 
complex permittivity $\varepsilon_{1}=\varepsilon_{0} \varepsilon_{\mathrm{r} 1}\left(1-\tan \delta_{1}\right)$, as shown in Fig. 1 . For space applications, the grounded dielectric layer may be honeycomb or another low mass qualified material. The proposed reflectarray element contains only one dielectric layer with the dipoles printed on both sides and one bonding layer. This results in a significant simplification of the manufacturing process with respect to that of reflectarrays based on the element with three stacked rectangular patches [3, 8], where at least three layers with printed elements and three bonding layers are required. In this paper, the dielectric layers chosen for operation frequencies in Ku-band are made of Diclad $880\left(\varepsilon_{\mathrm{r} 1}=\varepsilon_{\mathrm{r} 2}=\right.$ 2.17 and $\tan \delta_{1}=\tan \delta_{2}=0.0009$ ). The thicknesses of the dielectric layers are $h_{2}=0.127 \mathrm{~mm}$ and $h_{1}=3.175 \mathrm{~mm}$. The layers have been bonded using two sheets of $0.076 \mathrm{~mm}$ thick thermoplastic bonding film 6250 from Cuclad. All dipoles have been assumed to be $0.5 \mathrm{~mm}$ wide $\left(w_{2}=w_{1}\right)$. The centre of the dipoles of length $l_{2 i}(i=1,2)$ has been placed at the centre point of the reflectarray unit cell. The coplanar lateral dipoles of length $l_{1 i}$ have been symmetrically placed with respect to the central dipole of
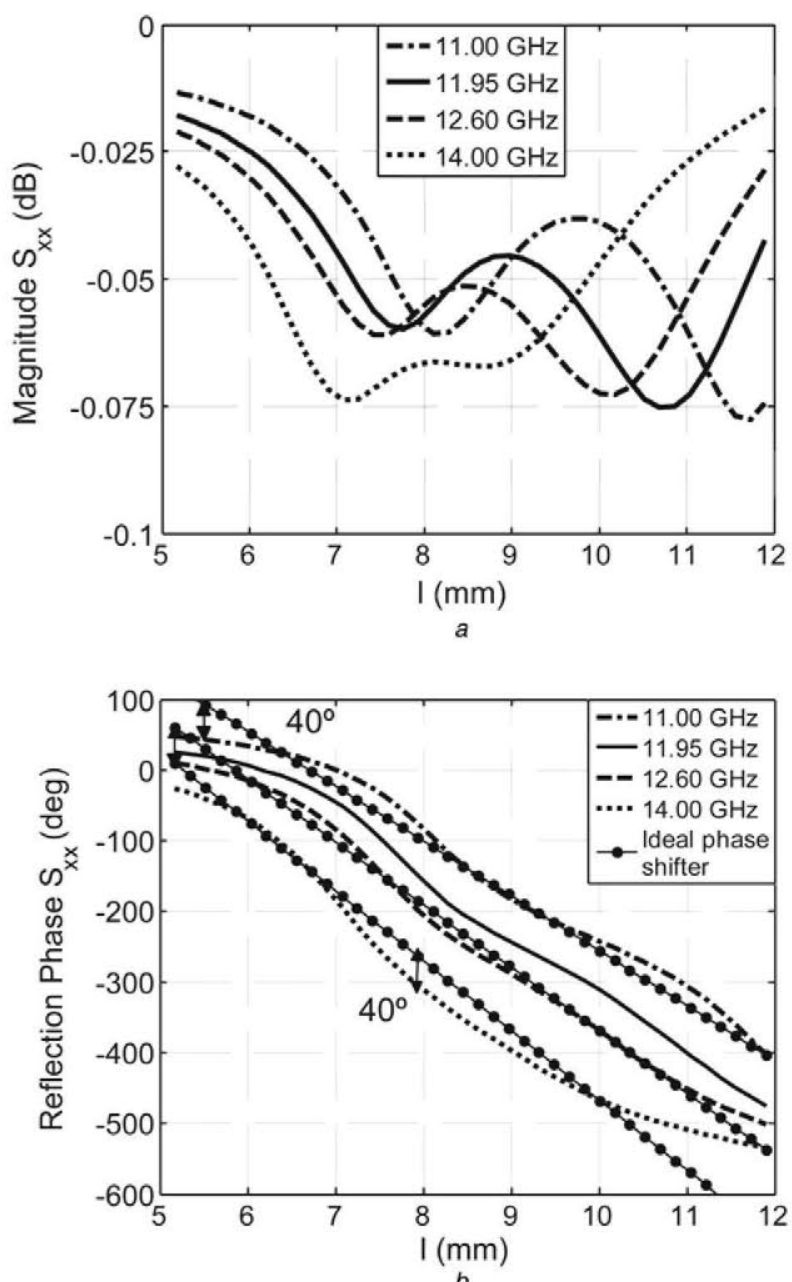

Fig. 2 Numerical results obtained by MoM-SD for the

$a$ Magnitude

$b$ Phase of $S_{x x}$ at different frequencies

Normal incidence is assumed. The phase curves are compared with those for an ideal phase shifter at three frequencies. Parameters: $a=b=12 \mathrm{~mm} ; l_{21}=$ $l_{22}=l ; l_{11}=l_{12}=0.63 l ; s_{1}=s_{2}=3.5 \mathrm{~mm} ; w_{1}=w_{2}=0.5 \mathrm{~mm} ; \varepsilon_{\mathrm{r} 1}=\varepsilon_{\mathrm{r} 2}=2.17$; $\tan \delta_{1}=\tan \delta_{2}=0.0009 ; h_{1}=3.175 \mathrm{~mm} ;$ and $h_{2}=0.127 \mathrm{~mm}$ length $l_{2 i}(i=1,2)$, which helps to reduce the cross-polarisation.

For the analysis and design of a reflectarray antenna based on the element of Fig. 1, each element of the antenna shown has been assumed to be embedded in an infinite periodic array of elements of the same size [2]. This is known as the local periodicity assumption [2], and it makes it possible to design reflectarray antennas within reasonable central processing unit times [1], even for very stringent requirements $[3,8]$. The validity of the local periodicity assumption is based on the fact that it leads to numerical results that show good agreement with experimental results $[2-4,8]$. A home-made software based on the method of moments in the spectral domain (MoMs-SD) [29] with multilayered Green's functions [30] has been implemented for the analysis of multilayered periodic structures containing the unit cell of Fig. 1 [31]. The accuracy of the home-made software has been checked by comparing their results with those obtained with the commercial software $\mathrm{CST}^{\circledR}$ (computer simulation technology) [32]. The home-made software has been used in the design of the reflectarray antenna under the local periodicity assumption since it has proven to be substantially faster than $\operatorname{CST}^{\circledR}[31]$. The lengths $l_{1 i}$ of coplanar lateral dipoles have been properly scaled with respect to the length $l_{2 i}$ of the central dipoles so that losses are minimised, and that variations in the slope of the phase curves are kept small within a phase range larger than $360^{\circ}[1,13]$. A parametric study has been carried out, and the scaling factor providing the most linear and smoothest phase curve within $500^{\circ}$ phase range with lowest losses has been found to be $l_{1 i}=0.63 l_{2 i}$ when $l_{21}=l_{22}$. In addition, the effect of the separation between dipoles on the magnitude and phase response has been studied, while maintaining the dimensions of the periodic unit cell $(a=12$ $\mathrm{mm}$ and $b=12 \mathrm{~mm}$ in Fig. 1). The results have shown that a separation $s_{1}=s_{2}=3.5 \mathrm{~mm}$ provides the lowest losses while keeping a linear phase response. This separation implies that the coplanar dipoles are equally spaced all over the reflectarray. Figs. 2 and 3 show the magnitude and phase of the co-polar elements of the Cartesian scattering matrices $S_{x x}$ and $S_{y y}$ at different frequencies when a plane wave impinges on the periodic cell of Fig. 1 at normal incidence (see Eqn. (1) of [31] for a definition of the Cartesian scattering matrix relating the Cartesian components of the electric field of the incident and reflected waves at the upper limit of a multilayered periodic structure). As can be seen, a smooth phase variation is obtained with a phase range larger than $400^{\circ}$ and losses better than $0.08 \mathrm{~dB}$ in a wide frequency band $(11-14 \mathrm{GHz})$ for both polarisations. To quantify the frequency dispersion of the proposed reflectarray elements, the phase errors have been evaluated at three frequencies $(11,12.6$ and $14 \mathrm{GHz})$ by comparing the simulated phase curves with the linear phase response corresponding to an ideal phase-shifter as defined in [1] (page 96) and [33], superimposed in Figs. $2 b$ and $3 b$. As shown in the figures, the maximum phase errors with respect to an ideal phase-shifter are $40^{\circ}$ for $X$-polarisation and $35^{\circ}$ for $Y$-polarisation for a range of phase-shift larger than $400^{\circ}$. These values of frequency dispersion from 11 to $14 \mathrm{GHz}$ ( $24 \%$ bandwidth) are quite good.

The sensitivity of the phase response to the angle of incidence has also been studied. Fig. 4 shows the phase curves at $11.95 \mathrm{GHz}$ for both polarisations at different angles of incidence up to $30^{\circ}$, which is the maximum incidence angle in the demonstrator reported in this paper. For this demonstrator, the incidence $\left(\theta=25^{\circ}, \phi=25^{\circ}\right)$, 
included in the figures, corresponds to elements near the reflectarray edge that contributes to the cross-polarisation most significantly. The maximum phase variations with the angle of incidence are $30^{\circ}$ for $X$-polarisation and $20^{\circ}$ for $Y$-polarisation, assuming dipoles shorter than $11.2 \mathrm{~mm}$. Note that for dipole lengths close to $12 \mathrm{~mm}$ there is a large variation with the angle of incidence for Y-polarisation, and also higher losses (not shown in the figure). To avoid this problem, the lengths of the dipoles will be limited to $11 \mathrm{~mm}$.

The results shown in Figs. 2-4 demonstrate that the reflectarray element of Fig. 1 has an excellent performance in terms of bandwidth, losses and low sensitivity to the angle of incidence.

\section{Design of reflectarray prototype}

A $40 \mathrm{~cm}$ reflectarray antenna has been designed to produce a focused beam in the direction $\theta_{b}=16.9^{\circ}$ and $\varphi_{b}=0^{\circ}$ at $11.95 \mathrm{GHz}$ for both linear polarisations. The reflectarray element characterised in the previous section has been used. The reflectarray is circular and consists of 861 elements arranged in a $33 \times 33$ grid with cell size $12 \mathrm{~mm} \times 12 \mathrm{~mm}$ (diameter of $396 \mathrm{~mm}$ ). The reflectarray is illuminated by a

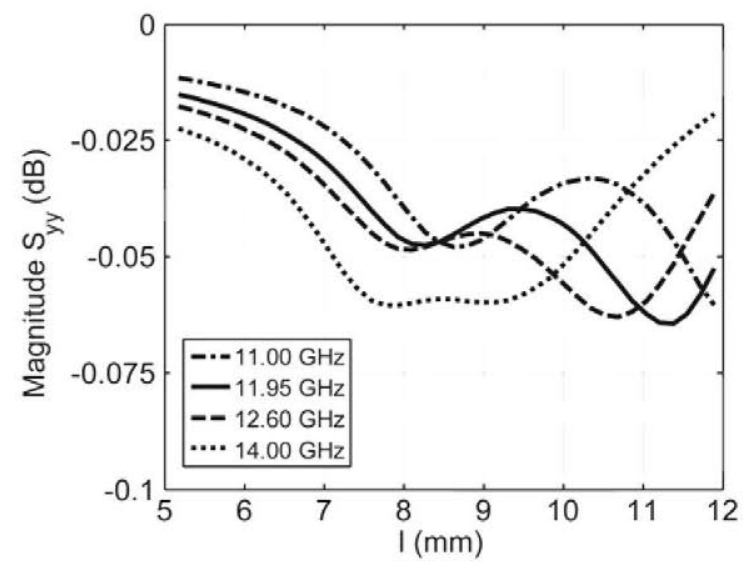

a

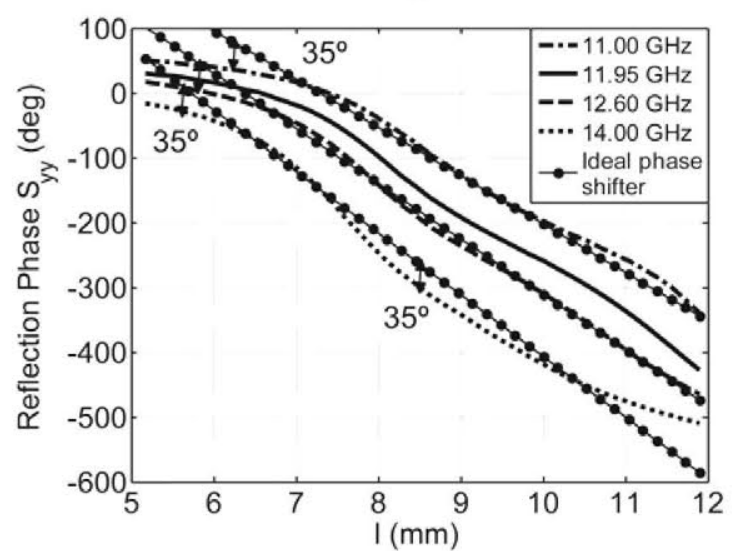

$b$

Fig. 3 Numerical results obtained by MoM-SD for the $a$ Magnitude

$b$ Phase of $S_{y y}$ at different frequencies

Normal incidence is assumed. The phase curves are compared with those for an ideal phase shifter at three frequencies. Parameters: $a=b=12 \mathrm{~mm} ; l_{21}=$ $l_{22}=l ; l_{11}=l_{12}=0.63 l ; s_{1}=s_{2}=3.5 \mathrm{~mm} ; w_{1}=w_{2}=0.5 \mathrm{~mm} ; \varepsilon_{\mathrm{r} 1}=\varepsilon_{\mathrm{r} 2}=2.17$; $\tan \delta_{1}=\tan \delta_{2}=0.0009 ; h_{1}=3.175 \mathrm{~mm} ;$ and $h_{2}=0.127 \mathrm{~mm}$

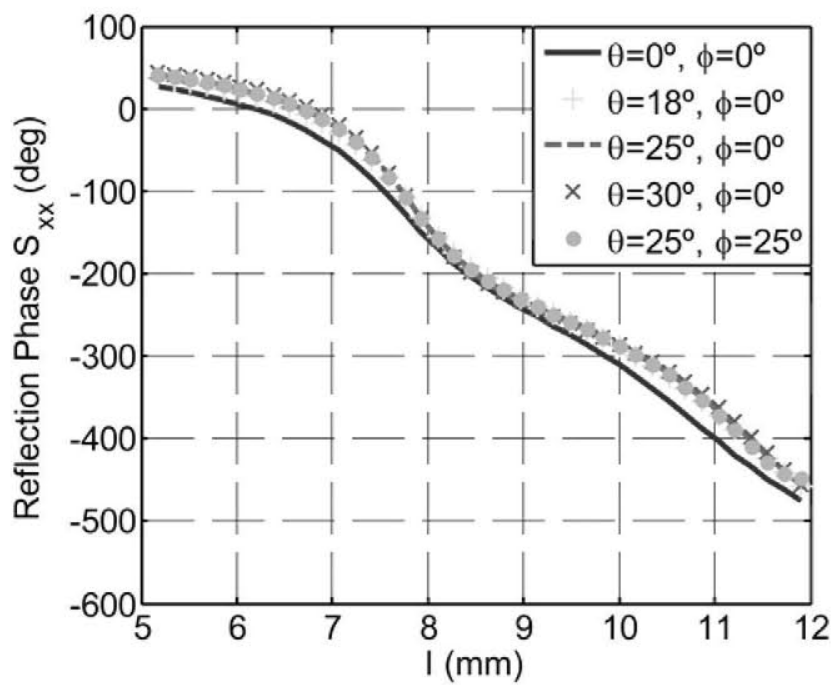

a

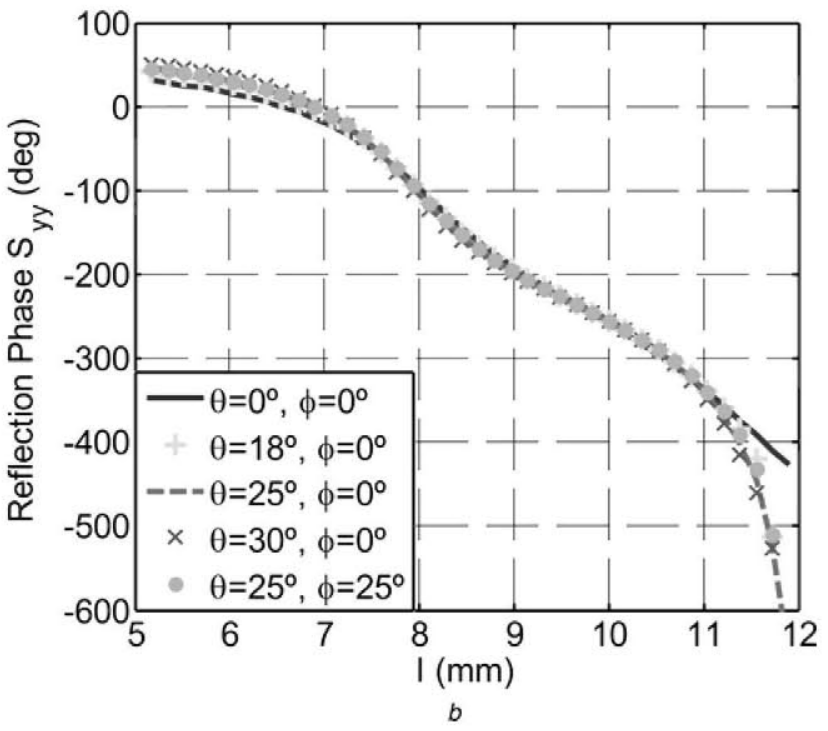

Fig. 4 Numerical results obtained by MoM-SD for the phase of $a S_{x x}$

$b S_{y y}$ at $11.95 \mathrm{GHz}$ for different incident angles

Parameters: $a=b=12 \mathrm{~mm} ; l_{21}=l_{22}=l ; l_{11}=l_{12}=0.63 l ; s_{1}=s_{2}=3.5 \mathrm{~mm}$; $w_{1}=w_{2}=0.5 \mathrm{~mm} ; \varepsilon_{\mathrm{r} 1}=\varepsilon_{\mathrm{r} 2}=2.17 ; \tan \delta_{1}=\tan \delta_{2}=0.0009 ; h_{1}=3.175 \mathrm{~mm} ;$ and $h_{2}=0.127 \mathrm{~mm}$

corrugated circular feed-horn with its phase centre located at coordinates $x=-193 \mathrm{~mm}, y=0 \mathrm{~mm}$ and $z=635 \mathrm{~mm}$ with respect to the centre of the reflectarray (see the coordinate system in Fig. $5 b$ ). Note that the angle between the $Z$-axis and the radiated beam has been chosen equal to the angle of incidence between the $Z$-axis and the line connecting the feed-horn and the reflectarray centre, which avoids the beam squint effect [34]. The feed-hom illuminates the edges of the reflectarray with a taper of $11 \mathrm{~dB}$ within a subtended angle of $31^{\circ}$.

For each element of the reflectarray, the lengths of each set of parallel dipoles for each polarisation have been adjusted separately to match the phase of $S_{x x}$ (for the $X$-polarisation, that is, for an incident electric field with the main component in the $X$-Z-plane) and $S_{y y}$ (for the $Y$-polarisation, that is, for an incident electric field with the main component along the $Y$-direction, and perpendicular to the $X-Z$-plane) to the required phase-shift that produces a collimated beam at $11.95 \mathrm{GHz}$. The lengths of the dipoles 

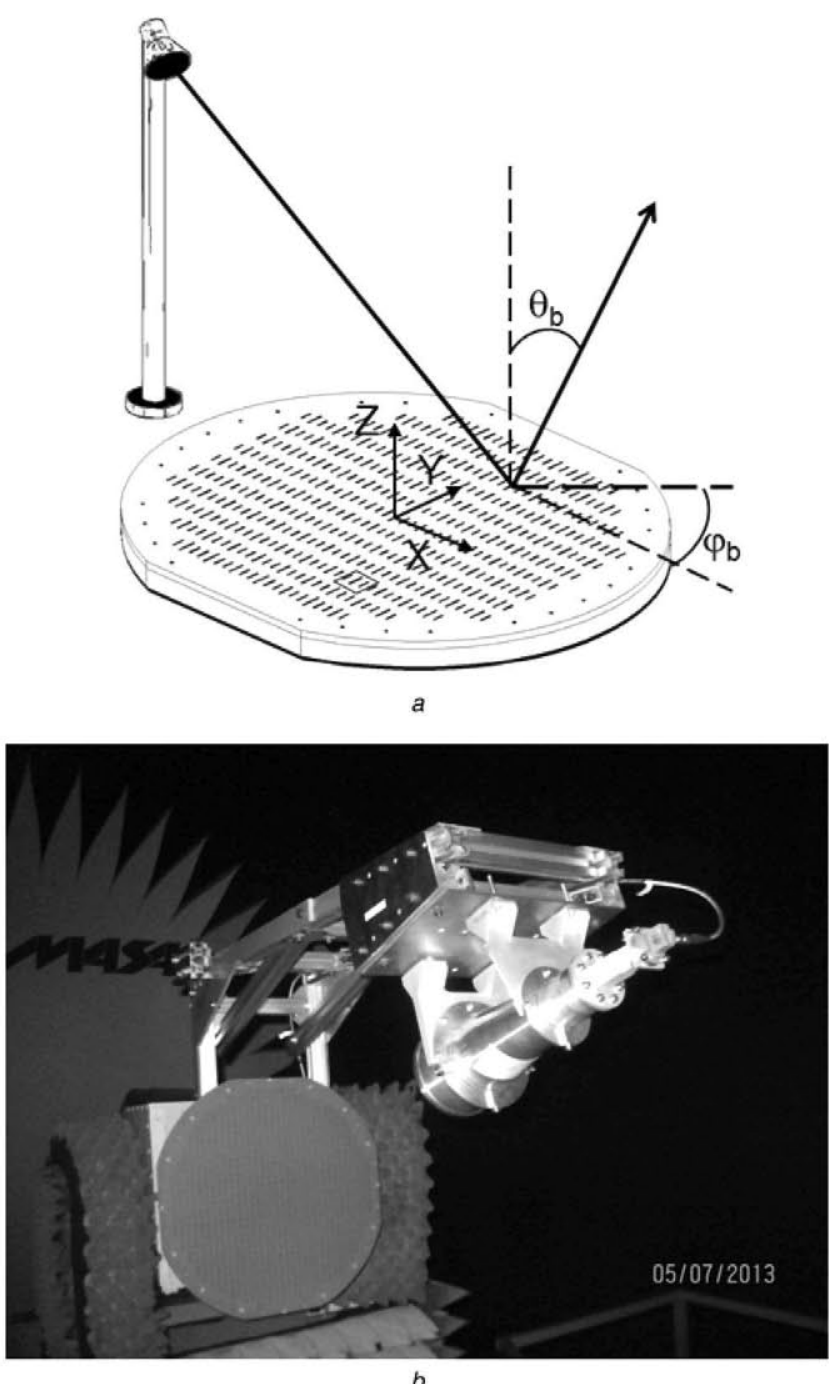

Fig. 5 Reflectarray antenna made of elements with two orthogonal sets of three parallel dipoles

$a$ Drawing including the reflectarray coordinates system

$b$ Photograph of the manufactured prototype

in each element have been adjusted by a zero finding routine which calls iteratively the implemented MoM-SD software, while taking into account the real angle of incidence associated to each element of the reflectarray.

The designed reflectarray has been manufactured and measured. Fig. $5 b$ shows a photograph of the manufactured prototype with 861 phase-shifting elements. Each of the two mask layouts of the reflectarray contains 2583 dipoles and a dielectric frame without dipoles. This dielectric frame is used to fix the antenna to the support structure with nylon screws.

\section{Measured and simulated radiation patterns}

The antenna prototype has been measured in a compact-range anechoic chamber, as shown in Fig. $5 b$. The measurements have been carried out for both polarisations ( $X$ and $Y$ ) in the azimuth and elevation planes. The numerical and experimental results obtained for the co-polar and cross-polar components of the radiated field at $11.95 \mathrm{GHz}$ are superimposed in Figs. 6 and 7. The numerical radiation patterns have been obtained from the tangential electric field reflected at each reflectarray element, which is

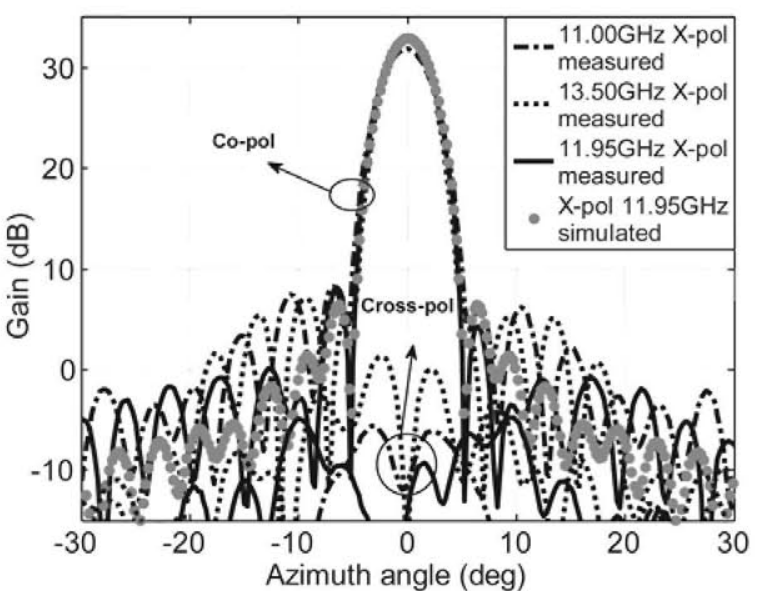

a

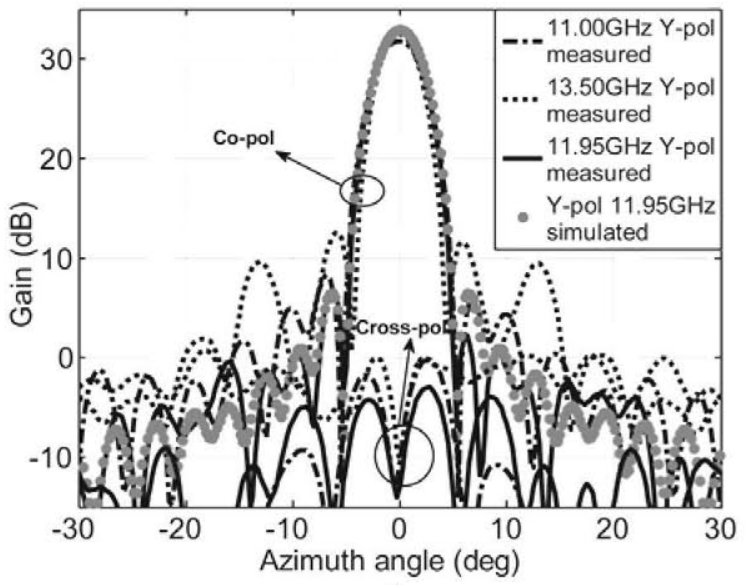

$b$

Fig. 6 Radiation patterns in the azimuth plane for

a $X$-polarisation

$b$ Y-polarisation at different frequencies

Solid lines (measurements) and dots and dashes (simulations) stand for the co-polar patterns. Dashed lines stand for the cross-polar patterns

computed by invoking the local periodicity condition, and by using the home-made MoM-SD software. In the computation of the fields radiated by the reflectarray, the dielectric frame without dipoles (see photograph of Fig. 5b) has been taken into account. The incident field on the reflectarray surface has been computed from the electric field on the aperture of the corrugated horn, obtained by a full-wave analysis of the horn using $\operatorname{CST}^{\circledR}$, by means of the angular spectrum of plane waves [35]. This approach means that the near-field is used to compute the incident field on each reflectarray cell.

Figs. 6 and 7 show an excellent agreement between the experimental and simulated co-polar radiation patterns in the main lobe and in the first side lobes for both polarisations at $11.95 \mathrm{GHz}$. The measured and simulated cross-polar patterns according to Ludwig's third definition [36] are also shown. The disagreements existing in Fig. 6 between the experimental and simulated cross-polar radiation patterns are attributed to the limitations of the compact test range used in the measurements, which is not accurate when the level of cross-polarisation is more than $30 \mathrm{~dB}$ below the maximum of radiation, as it happens in the results of Fig. 6. Since the experimental and theoretical results show levels of cross-polarisation that are more than $30 \mathrm{~dB}$ below the co-polar maximum, an accurate measurement of these levels of cross-polarisation would 
require a three-dimensional data acquisition experimental setup that uses a planar or spherical near-field system with a high cross-polar discrimination probe. The simulated cross-polar radiation patterns in the elevation plane are not shown in Fig. 7 because their value is lower than $-30 \mathrm{dBi}$ for both polarisations (this is to be expected since the elevation plane is a symmetry plane of the antenna). The small asymmetries observed in the measured co-polar patterns in azimuth (Fig. 6) and the non-zero values of the measured cross-polar radiation in the elevation plane (Fig. 7) are attributed to manufacturing tolerances, which results in small physical asymmetries of the prototype.

The maximum gain measured at $11.95 \mathrm{GHz}$ for both polarisations is around $32.94 \mathrm{~dB}$, which represents a $60 \%$ antenna efficiency when the dielectric frame without dipoles is taken into account (the theoretical maximum gain is $35.36 \mathrm{~dB}$ ). Note that the antenna efficiency would increase to $71 \%$ if the dielectric frame without dipoles were not considered (the theoretical maximum gain would be $33.9 \mathrm{~dB}$ in this case). The largest side lobes are roughly $20 \mathrm{~dB}$ below the maximum of radiation (and $25 \mathrm{~dB}$ below the maximum at the design frequency of $11.95 \mathrm{GHz}$ ), and the cross-polar radiation is at least $30 \mathrm{~dB}$ below the co-polar gain in both polarisations for all the frequencies.

Figs. 6 and 7 also show the experimental radiation patterns for the two polarisations at different frequencies in the azimuth and elevation planes, respectively. In both
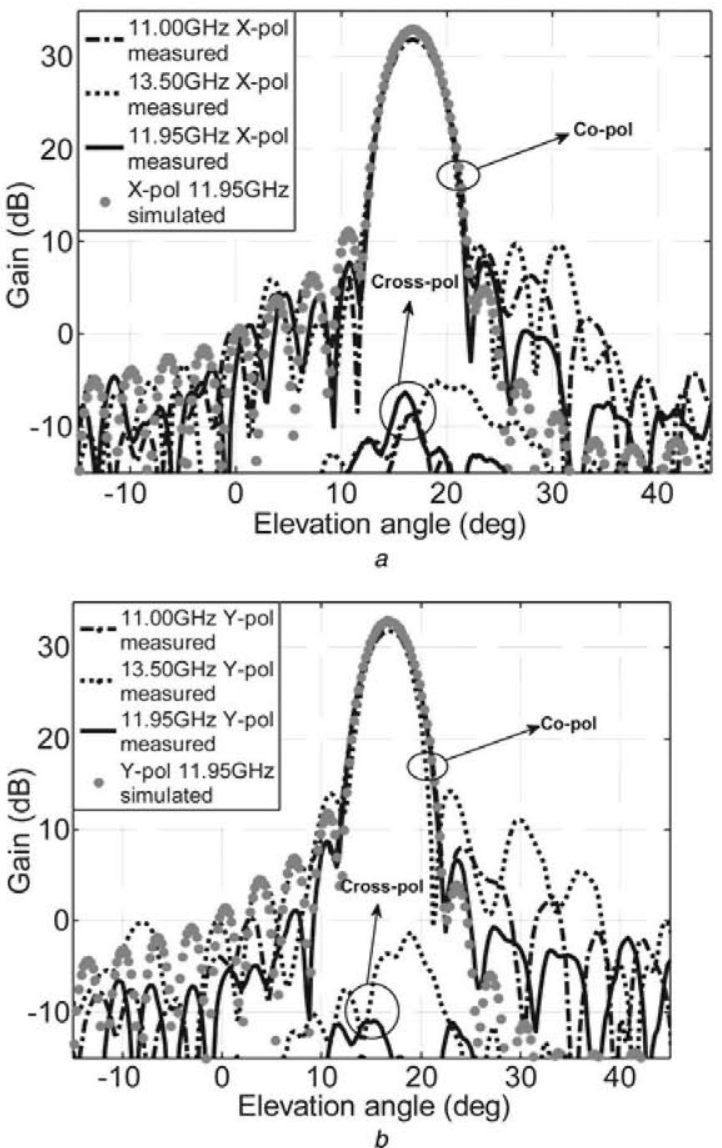

Fig. 7 Radiation patterns in the elevation plane for

a $X$-polarisation

$b Y$-polarisation at different frequencies

Solid lines (measurements) and dots and dashes (simulations) stand for the co-polar patterns. Dashed lines stand for the cross-polar patterns polarisations, the maximum gain variation is $<1 \mathrm{~dB}$, the side lobe level is roughly kept $20 \mathrm{~dB}$ below the maximum and the maximum of cross-polar radiation is about $30 \mathrm{~dB}$ below the main beam co-polar radiation in the range of frequencies from 11 to $13.5 \mathrm{GHz}$. Note that the side lobe level is lower in the case of the $X$-polarisation in both planes. The side lobe level tends to increase with frequency, as shown in Figs. 6 and 7. However, the antenna gain remains very stable in the whole range of frequencies from 11 to $14.5 \mathrm{GHz}$, as shown in Fig. 8. According to these results, we can conclude that the $1 \mathrm{~dB}$ bandwidth is $27 \%$ for both polarisations, even though small pattern distortions are observed above $13.5 \mathrm{GHz}$. Note that this bandwidth performance has been achieved for the antenna designed at a single frequency $(11.95 \mathrm{GHz})$ because of the broadband behaviour of the reflectarray element, and because of the moderate size of the reflectarray antenna. However, the bandwidth could be improved in larger antennas by using an optimisation routine that enforces the required phase at different frequencies as in some previous works $[3,6,8]$.

The maximum level of cross-polarisation in the angular interval corresponding to a $3 \mathrm{~dB}$ beamwidth has also been plotted in Fig. 8 in the whole range of frequencies for both polarisations. In general, the level of cross-polarisation is lower for $X$-polarisation, except in the vicinity of $12.9 \mathrm{GHz}$. This fact, also corroborated by the measurements shown in Fig. 6, can be explained because in the $X$-polarisation the cross-polarisation is only produced by the field scattered by the dipoles, when they are excited under oblique incidence, being the contribution from the feed-horn negligible. On contrary, the contributions from the feed-horn and from the dipoles are added to generate the overall cross-polar radiation in $Y$-polarisation. In some particular cases, the cross-polarisation produced by the horn in $Y$-polarisation can be cancelled with that produced by the dipoles, as it happens at $12.9 \mathrm{GHz}$ in Fig. 8.

\section{Design of reflectarray with different beams for each polarisation}

In this section, we demonstrate the capabilities of the antenna element introduced in Section 2 to provide independent beam forming in each polarisation, while maintaining a high

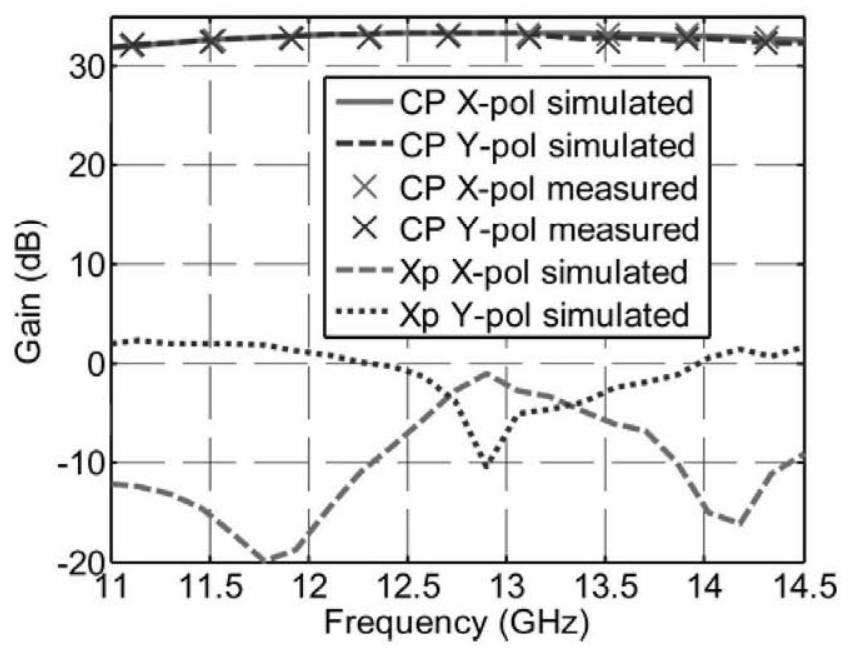

Fig. 8 Simulated and measured maximum values of the co-polar and cross-polar radiation in the main beams of the radiation patterns as a function of frequency for the two polarisations 
performance in bandwidth, efficiency and cross-polarisation. For that purpose, a $40 \mathrm{~cm}$ reflectarray antenna with the same geometrical dimensions of the antenna of Sections 3 and 4 has been designed to produce two pencil beams at $11.95 \mathrm{GHz}$, one in the direction $\theta_{b}=13^{\circ}$ and $\varphi_{b}=0^{\circ}$ for $X$-polarisation, and the other in the direction $\theta_{b}=20^{\circ}$ and $\varphi_{b}=0^{\circ}$ for $Y$-polarisation.

Figs. 9 and 10 show the simulated radiation patterns for the two polarisations at different frequencies in the azimuth and elevation planes, respectively. In both polarisations, the maximum gain variation is $<1 \mathrm{~dB}$, the side lobe level is roughly kept $20 \mathrm{~dB}$ below the maximum and the maximum cross-polar radiation is about $35 \mathrm{~dB}$ below the main beam co-polar radiation in the range of frequencies from 11 to 13 GHz. In Fig. 10, we can observe a slight beam squint inferior to $0.5^{\circ}$. Despite the angle between the $Z$-axis and the radiated beam does not coincide with the angle of incidence between the $Z$-axis and the line connecting the feed-horn and the reflectarray centre, the slight beam squint is almost negligible (smaller than $0.5^{\circ}$ ). Note that the maximum gain at $11.95 \mathrm{GHz}$ is $32.57 \mathrm{~dB}$ for $X$-polarisation and $32.17 \mathrm{~dB}$ for $Y$-polarisation, which represents a 73 and $67 \%$ antenna efficiency, respectively (when the dielectric frame is not taken into account). Although the side lobe

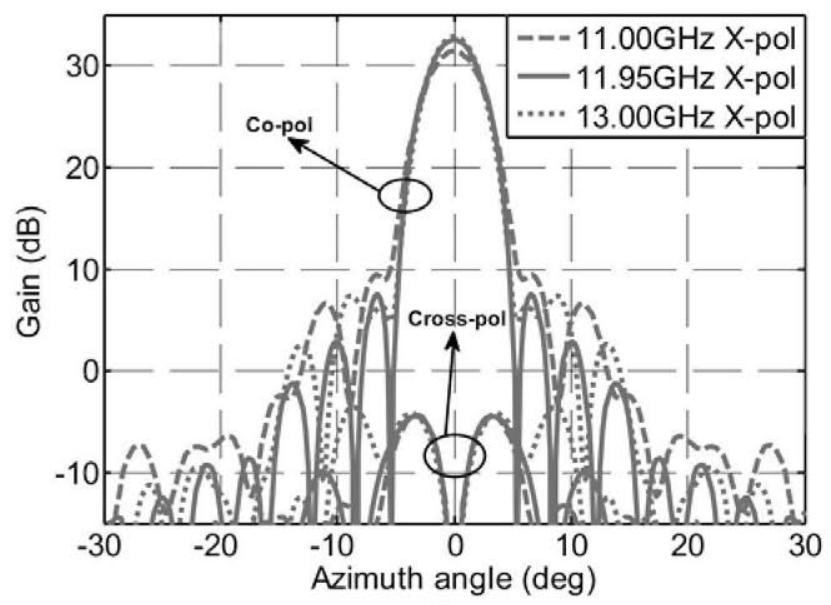

a

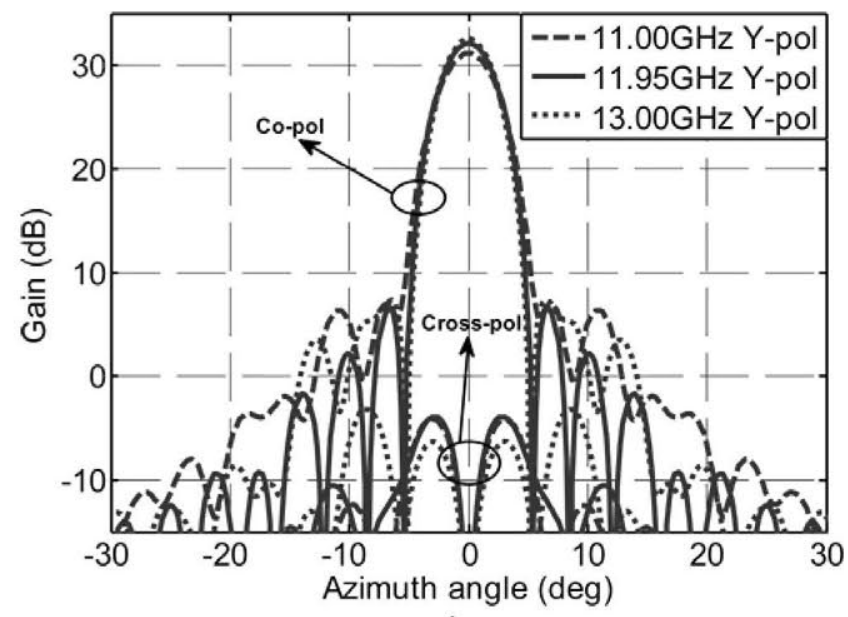

$b$

Fig. 9 Simulated radiation patterns for the antenna with separate beams in each polarisation. The patterns are plotted in the azimuth plane for

a $X$-polarisation

b $Y$-polarisation at different frequencies

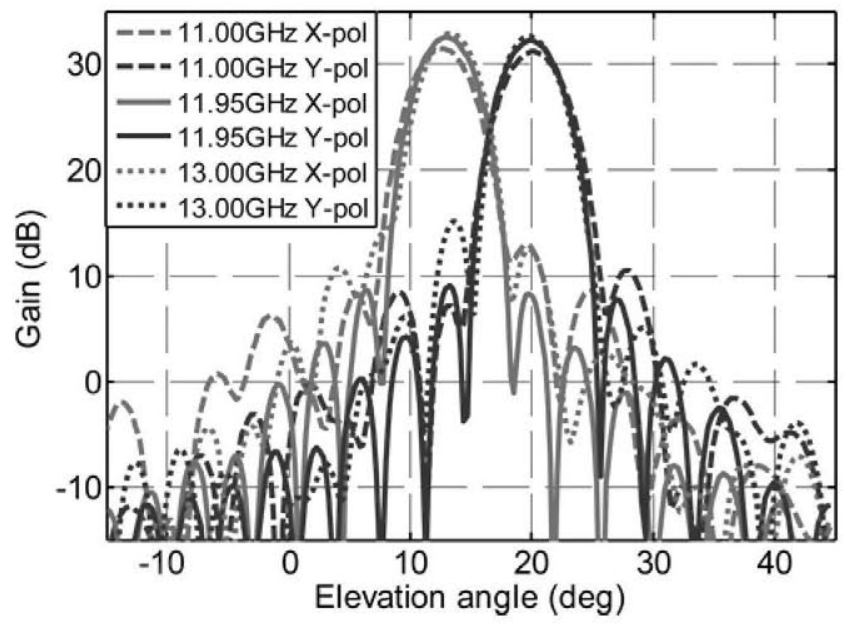

Fig. 10 Simulated radiation patterns for the antenna with separate beams in each polarisation

Patterns are plotted in the elevation plane for $X$-polarisation and $Y$-polarisation at different frequencies

level tends to increase as the frequency is changed from the design frequency $(11.95 \mathrm{GHz})$, as shown in Fig. 10, the antenna gain and the maximum cross-polarisation remain stable in the whole range of frequencies from 11 to 14.5 $\mathrm{GHz}$, as shown in Fig. 11. According to these results, we can conclude that the $1 \mathrm{~dB}$ bandwidth is $27 \%$ for both polarisations, although small pattern distortions are observed above $13 \mathrm{GHz}$. Once again, these results demonstrate the very high performance of the proposed antenna element in terms of bandwidth, efficiency and cross-polarisation, even though separate beams are radiated in each polarisation.

Whereas the cross-polar levels in the previous prototype were, in general, higher for $Y$-polarisation, the cross-polar levels in the present antenna are lower than $-3 \mathrm{dBi}$ with very similar values for both polarisations, as shown in Fig. 11. We have studied this behaviour in detail and we have found that in this case the cross-polar levels are reduced in $Y$-polarisation, because the feed contribution to

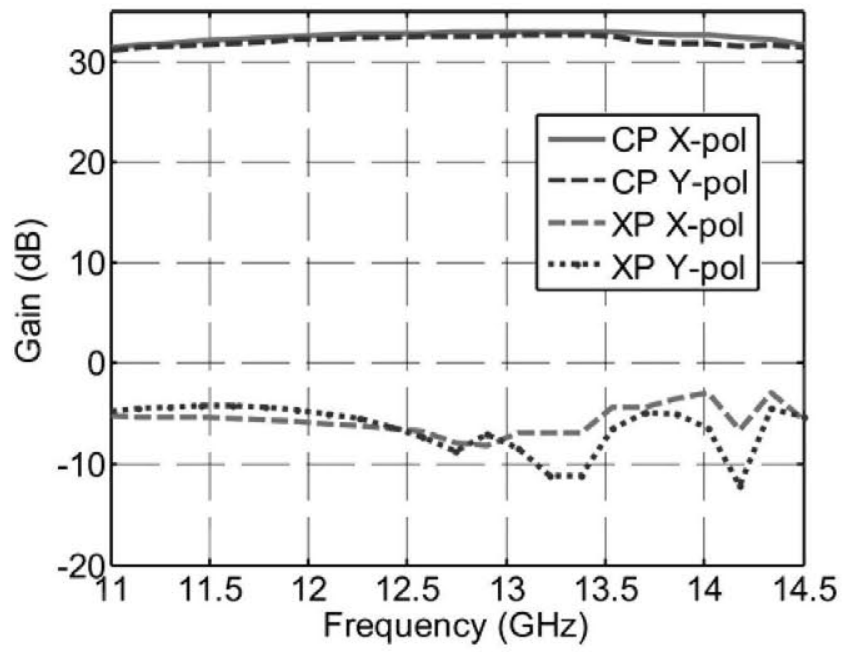

Fig. 11 Simulated maximum values of the co-polar and cross-polar radiation in the main beams of the radiation patterns as a function of frequency for the two polarisations

Results plotted correspond to the antenna with separate beams in each polarisation 
the cross-polarisation partially cancels the element scattering contribution to the cross-polarisation. In the case of the $X$-polarisation, the feed contribution to the cross-polarisation is negligible with respect to the element scattering contribution, and therefore does not have any effect on the cross-polarisation level of the antenna. Therefore the cross-polarisation levels are of the same order in both Figs. 8 and 11.

\section{Conclusions}

A broadband dual-polarisation reflectarray antenna with improved cross-polarisation properties has been introduced in this paper. The reflectarray element is made of two orthogonal sets of three coplanar parallel dipoles. Each set of parallel dipoles is used for adjusting the required phase-shift in each polarisation. The reflectarray element has shown a linear and smooth behaviour of the phase curves and low losses (lower than $0.08 \mathrm{~dB}$ ) in a wide interval of frequencies (from 11 to $14 \mathrm{GHz}$ ). The flexibility provided by the independent adjustment of the phase for each polarisation makes this reflectarray element suitable for the radiation of different beams in each polarisation, as required in some specific applications [3, 28].

A $40 \mathrm{~cm}$ focused beam reflectarray antenna has been designed, manufactured and tested. The results show a $27 \%$ antenna bandwidth for a gain variation of $1 \mathrm{~dB}$ and cross-polar radiation at least $30 \mathrm{~dB}$ below the co-polar radiation within a $3 \mathrm{~dB}$ beamwidth for both polarisations. The antenna efficiency is $60 \%$ when the structural frame of the antenna prototype is considered, and can be increased up to $71 \%$ as the size of this frame is reduced.

The results presented in this paper show that the proposed reflectarray element is suitable to design reflectarrays for dual polarisation with high performance in terms of bandwidth, antenna efficiency and cross-polarisation. For a further bandwidth improvement in larger antennas, the dimensions of all the dipoles can be optimised by using an optimisation routine that enforces the required phases at different frequencies in one band $[3,6]$ or in several frequency bands as in the case of transmit-receive antennas [8].

\section{Acknowledgments}

This work has been supported by the Spanish Ministry of Science and Innovation (projects CICYT TEC2010-17567, TEC2013-43345-P and Consolider Ingenio 2010 CSD2008-00066), by the European Space Agency under ESTEC contract no. 4000106334 and by the 'Junta de Andalucía' (project P12-TIC-1435).

\section{References}

1 Huang, J., Encinar, J.A.: 'Reflectarray antemnas' (Wiley-IEEE Press, New Jersey, 2007)

2 Pozar, D.M., Targonski, S.D., Syrigos, H.D.: 'Design of millimeter wave microstrip reflectarray', IEEE Trans. Antennas Propag., 1997, 45, pp. 287-296

3 Encinar, J.A., Datashvili, L., Zornoza, J.A., et al.: 'Dual-polarization dual-coverage reflectarray for space applications', IEEE Trans. Antennas Propag., 2006, 54, pp. 2828-2837

4 Encinar, J.A.: 'Design of two-layer printed reflectarrays using patches of variable size', IEEE Trans. Antennas Propag., 2001, 49, pp. 1403-1410

5 Bozzi, M., Germani, S., Perregrini, L.: 'Performance comparison of different element shapes used in printed reflectarrays', IEEE Antennas Wirel. Propag. Lett., 2003, 2, pp. 219-222

6 Encinar, J.A., Zornoza, J.A.: 'Broadband design of three-layer printed reflectarrays', IEEE Trans. Antennas Propag., 2003, 51, pp. 1662-1664
7 Carrasco, E., Encinar, J.A., Barba, M.: 'Bandwidth improvement in large reflectarrays by using true-time-delay', IEEE Trans. Antennas Propag., 2008, 56, pp. 2496-2503

8 Encinar, J.A., Arrebola, M., de la Fuente, L.F., Toso, G.: 'A transmitreceive reflectarray antenna for direct broadcast satellite applications', IEEE Trans. Antennas Propag., 2011, 59, pp. 3255-3264

9 Menzel, W., Jiang, L., Dieter, S.: 'Folded reflectarray antenna based on a single layer reflector with increased phase angle range'. Proc. of the Third European Conf. on Antennas and Propagation, EuCAP 2009, Berlin, Germany, March 2009, pp. 2757-2760

10 Li, L., Chen, Q., Yuan, Q., et al.: 'Novel broadband planar reflectarray with parasitic dipoles for wireless communication applications', IEEE Antennas Wirel. Propag. Lett., 2009, 8, pp. 881-885

11 Carrasco, E., Barba, M., Encinar, J.A., Arrebola, M., Rossi, F., Freni, A.: 'Design, manufacture and test of a low-cost shaped-beam reflectarray using a single layer of varying-sized printed dipoles', IEEE Trans. Antennas Propag., 2013, 61, pp. 3077-3085

12 Sayidmarie, K.H., Bialkowski, M.E.: 'Investigations into unit cells offering and increased phasing range for single-layer printed reflectarrays', Microw. Opt. Technol. Lett., 2007, 50, (4), pp. 1028-1032

13 Sayidmarie, K.H., Bialkowski, M.E.: 'Fractal unit cells of increased phasing range and low slopes for single-layer microstrip reflectarrays', IET Microw. Antennas Propag., 2011, 5, pp. 1371-1379

14 Chaharmir, M.R., Shaker, J., Cuhaci, M., Ittipiboon, A.: 'A broadband reflectarray antenna with double square rings', Microw. Opt. Technol. Lett., 2006, 48, (7), pp. 1317-1320

15 Chaharmir, M.R., Shaker, J.: 'Broadband reflectarray with combination of cross and rectangle loop elements', Electron. Lett., 2008, 44, pp. 658-659

16 Chaharmir, M.R., Shaker, J., Legay, H.: 'Broadband design of a single layer large reflectarray using multi cross loop elements', IEEE Trans. Antennas Propag., 2009, 57, pp. 3363-3366

17 Moustafa, L., Gillard, R., Peris, F., Loison, R., Legay, H., Girard, E. 'The Phoenix cell: a new reflectarray cell with large bandwidth and rebirth capabilities', IEEE Antennas Wirel. Propag. Lett., 2011, 10, pp. $71-74$

18 Zhou, M., Sørensen, S.B., Kim, O.S., et al.: 'The generalized printed optimization technique for printed reflectarrays', IEEE Trans. Antennas Propag., 2014, 62, pp. 1690-1700

19 Florencio, R., Boix, R.R., Encinar, J.A.: 'Comparative study of reflectarrays based on cells with three coplanar dipoles and reflectarrays based on cells with three stacked patches'. Proc. Sixth Edition EUCAP, Prague, Czech Republic, March 2012

20 Cadoret, D., Marnat, L., Loison, R., Gillard, R., Legay, H., Salomé, B.: 'A dual linear polarized printed reflectarray using slot loaded patch elements'. Proc. of the Second European Conf. on Antennas and Propagation, EuCAP 2007, Edinburgh, UK, November 2007

21 Chaharmir, M.R., Shaker, J., Gagnon, N., Lee, D.: 'Design of broadband single layer dual-band large reflectarray using multi open loop elements', IEEE Trans. Antennas Propag., 2010, 58, pp. 2875-2883

22 Shaker, J., Pike, C., Cuhaci, M.: 'A dual orthogonal Cassegrain flat reflector for Ka-band application', Microw. Opt. Technol. Lett., 2000, 24, pp. 7-11

23 Hasani, H., Peixeiro, C.: 'Dual-band, dual-polarized microstrip reflectarray antenna in $\mathrm{Ku}$ band'. 2012 Loughborough Antennas and Propagation Conf. (LAPC), 12-13 November 2012, pp. 1-3

24 Perruisseau-Carrier, J.: 'Dual-polarized and polarization-flexible reflective cells with dynamic phase control', IEEE Trans. Antennas Propag., 2010, 58, (5), pp. 1494-1502

25 Moghadas, H., Daneshmand, M., Mousavi, P., Chaharmir, M.R. Shaker, J.: 'Orthogonally-polarized dual-band MEMS-tunable double-slotted unit cell for reflectarray applications'. 2012 IEEE Antennas and Propagation Society Int. Symp. (APSURSI), 8-14 July 2012, pp. 1-2

26 Makdissy, T., Gillard, R., Fourn, E., Girard, E., Legay, H.: 'Phase-shifting cell loaded with variable capacitances for dual linearly polarised reflectarrays', Electron. Lett., 2012, 48, (21), pp. 1319-1320

27 Makdissy, T., Gillard, R., Fourn, E., Girard, E., Legay, H.: 'Phase-shifting cell for dual linearly polarized reflectarrays with reconfigurable potentialities', IEEE Antennas Wirel. Propag. Lett., 2014, 13, pp. 11-14

28 Rengarajan, S.R.: 'Reflectarrays of rectangular microstrip patches for dual-polarization dual-beam radar interferometers', Prog. Electromagn. Res., 2013, 133, pp. 1-15

29 Mittra, R., Chan, C.H., Cwik, T.: 'Techniques for analyzing frequency selective surfaces - a review', Proc. IEEE, 1988, 76, pp. 1593-1615

30 Mesa, F., Marqués, R., Horno, M.: 'A general algorithm for computing the bidimensional spectral Green's dyads in multilayered complex bianisotropic media: the equivalent boundary method', IEEE Trans. Microw. Theory Tech., 1991, 39, pp. 1640-1649 
31 Florencio, R., Boix, R.R., Carrasco, E., Encinar, J.A., Losada, V.: 'Efficient numerical tool for the analysis and design of reflectarrays based on cells with three parallel dipoles', Microw. Opt. Technol. Lett., 2013, 55, pp. 1212-1216

32 CST Microwave Studio ${ }^{\circledR}$. Available at http://www.cst.com, accessed

33 Florencio, R., Boix, R.R., Carrasco, E., Encinar, J.A., Barba, M., Perez-Palomino, G.: 'Broadband reflectarrays made of cells with three coplanar parallel dipoles', Microw. Opt. Technol. Lett., 2014, 56, pp. $748-753$
34 Targonski, S.D., Pozar, D.M.: 'Minimization of beam squint in microstrip reflectarrays using an offset feed'. Antennas and Propagation Society Int. Symp., 1996. AP-S. Digest, July 1996, pp. $1326-1329$

35 Clemmow, P.C.: 'The plane wave spectrum representation of electromagnetic fields' (Wiley-IEEE Press, New Jersey, 1996)

36 Ludwig, A.C.: 'The definition of cross-polarization', IEEE Trans. Antennas Propag., 1973, 21, pp. 116-119 